\title{
Study on the Product Symbol System for Brand R\&D
}

\author{
Qin Wang \\ School of Mechanical and Electronic Engineering, Dongguan University of Technology \\ Dongguan 523808, China
}

Tel: 86-769-2307-8216 E-mail:wangqinjaney@126.com

\begin{abstract}
The development orientations of the product brand design in the market are toward conception and symbolization. Based on the design semantics and the product semantics, the design thoughts and levels of the product symbol system was studied in the article. The results of the article could help design personnel in enterprises to more deeply understand the importance of the strategic development of new products and the effective application of the product symbol system.
\end{abstract}

Keywords: Product symbol system, Interaction design, Cultural semantics, Symbolization

\section{Introduction}

The word of "semiotics" was first proposed by the Switzerland linguist Sauaure and American philosopher and pragmatics Pierce. Though the academe has not formed complete uniform definition of the symbol, but most scholars thought that the symbol could be regarded as the material carrier of social information, and it could load certain information and transfer thoughts, and it could be the signified things such as English letter, Arabic numerals and finger alphabet for special things, and it could be the symbolization of certain abstract concept. Three necessary characters of the symbol include that (1) symbols must be matters, (2) symbols must transfer the information which is different to the carrier in nature, i.e. symbols must represent other things, (3) symbols must transfer a sort of social information which are established by the public not by the individual. Above opinion organically combine the material character of the symbol with the thought character, and it could enlighten people to understand the deep semiotics and human connotation of the symbol.

There are methods to classify the symbols. American semeiotic scientist and esthetician Suzanne Lenglen classified symbols as "deduction symbols" and "simultaneous symbols". She thought that the former meant the language symbols specially, and the latter was a sort of presentational symbol (i.e. a sort of complex symbol system, or the direct combination of color, line and subject, or the vision image directly combined by parabolic and poetic languages, and it is presented by the total imago, and it is also called as "presentational symbol" or "symbol of art"). According to the interactive relationship among three factors (agent, objective and explanation) of symbols, American philosopher Pierce established the "symbols classified method of three in one", and the core types included icon, index and symbol. According to the characters of "signified and signifier" of symbols, some scholars put forward five types including omen symbol, signified symbol, induction symbol, language symbol and substitute symbol (Xu, 2008).

Though the extension of symbol is wide, but in practical application, the symbols in the product design are mainly used to connect the objective and consumers to realize the interactive communication by the forms such as information and culture. The design elements and the basic measures are regarded as the symbols to increase and diffuse the information cultures of products and establish the innovational brands through integrating these elements.

Human researches and practices indicated that symbolized thinking and behaviors were the most representative characters in human life, and all cultural forms were forms of symbol, and the symbol design held the balance in the whole human social evolvement. Just as Cassirer said, "symbol thinking overcomes human natural inertia, and endows human a new ability to continually update the world". In the days that the science and technology develop quickly, the products updating speed is so quick, and the consumption demand contents change fundamentally, the establishment of the utilization of the design semeiotics based on product semeiotics and the innovational design of the product symbol system will help enterprises to effectively develop the new products, establish special brands and enhance the market competitive forces of products.

Based on above contents, the application of the product symbol system in the innovational design of brand will be deeply analyzed from the concept and levels of the product symbol system in the article.

\section{Concept analysis of the product symbol system design}

Usually, product symbols are some design elements attached on the modeling of product, or the icons or visualized operation indications which represent special brand. How ever, the interactive integration of multiple subject knowledge 
will widen the view of product design and enrich the methods of product design. Letter, language, Chinese traditional lines, various propagation forms in the nature, and many indicative expressions with designers' emotions and totems deduced from abstracted distortion all can be integrated into the product design process as the symbol elements.

But the design of modern industrial products can not only stay on the simple level of two-dimensional symbol element, and the single two-dimensional level design has not fulfill enterprises' requirements to research and develop strategic brand for a long time, and only the multi-dimensional product symbol system design can really fulfill the demands of the future market and establish special brand.

Generally speaking, the product symbol system includes three dimensions, i.e. the technical material dimension (including technology and materials), the semantics dimension of the technology object (configuration), and the syntactics dimension of technical function (composing). With the alterable freedom degrees of these three dimensions, the product can be turned into the design object, and only the product with these three dimensions, the product can acquire the symbol character. The organizer of the brand must completely grasp these three dimensions and exactly understand the referential function of the product symbol combining with the knowledge about the semeiotics and syntactics to establish applied product symbol system with bright features combining with consumers' psychological and sensible appeals.

\section{Level analysis of the product symbol system design}

The levels of the whole product symbol system directly influence the design of the innovational brand. From following aspects, the different levels of the product symbol system will be analyzed, and corresponding opinions will be proposed.

\subsection{Interaction layer analysis of the symbol system}

As the modern brand product design is required to not only fulfill consumers' demands from the appearance and function, but consider consumers sensible demands. Therefore, the design and R\&D of the product symbol system should comprehensively consider the appearance, function, consumers' minds, ergonomic engineering, the design concept, emotion and cultures which are easily identified and trusted by consumers. As the agent of the bilateral communication between designers and consumers, the operation instruction symbols in the product should make consumers exactly know how to operate and use the product, and consider the cultural differences in different regions and the combination of the culture and technology, i.e. consider the three dimensions of the system. The interaction is represented in that the design should take the consumer as the design topic, and take certain information and culture as the core of the whole product symbol design system to realize the mutual communication between consumers and products. The good interaction of the symbol system requires that the interaction could be integrated into the hard and soft sensibility. The structure relation of the interactive symbol system proposed in the article is seen in Figure 1.

\subsubsection{Image vision symbol interaction of the system}

The vision information of innovational product includes three aspects such as configuration, color and materials (Mao, 2008). The image interaction of the system in the hard and soft design innovations could directly leave good effects to consumers. Taking the automatic smile shutter mode of Sony digital camera as the example, based on the face automatic focus technology of the camera, users can select this mode and press the shutter button of the camera when screening portrait, Cyber-shot will look for the smile in the screening process and make the photo when detecting the smile face. Smile shutter mode can also distinguish adult's smile and child's smile, and the degree of the smile can be enacted as low, middle and high. Thus, the camera could automatically catch the most wonderful instant in the life (seen in Figure 2). The "happy face retouch" function of Sony digital camera could turn the suffering face into the happy face by the digital processing (seen in Figure 3). The mode which could realize the function innovation of product could accord with human taste habits and fulfill human mental appeals. As the bright point of the brand product, the smile face which is a sort of symbol with apperception and emotion is liked deeply by consumers.

\subsubsection{Sound hearing symbol interaction of the system}

The most natural, fluent, convenient information communication mode is language, and the most abundant and exact signification in the semeiotics is the sound symbol. The researches of ergonomics indicated that the hearing information and the vision information could offer human intensive third dimension and proximity sensibility simultaneously, but the detection speed of the hearing information is quicker than the detection speed of the vision singles. Generally speaking, the sound contains language, music and sound effect. As the designer completely grasps the sound symbol system, he could process the sounds emitted by the product based on art and sensibility to make the product could transfer certain special information to consumers through the ergonomic interaction. For example, the single tone of short message warning tone and the incoming tone of Nokia phone have been the bright point of the Nokia brand culture because the "single tone" accords with human language habits and hearing habits. The special rings have been the special symbol of Nokia and the diffusion media of the brand of Nokia. 


\subsubsection{Behavior touching symbol interaction of the system}

The handle is consumers' feeling when they directly contact the materials and configuration of the product. Good operation and control feeling could maximally exert the function of the product (Cheng, 2008 \& Cupar, 2006). The design of the feeling sense symbol system closing the body has been the development direction of the design, so the establisher and developer of the brand should be good at combining the correlative principles of ergonomics to create the product symbol system according with consumers' physiological and psychological demands. For the Wii game console brand established by Japan Nintendo Corporation, as the optimal exterior fittings of the Wii game console, Wii Fit can not only offer various games, but do various fitting sports for consumers. The Wii game console adopts revolutionary handle operation and control mode to change the key-press control mode in the past, and innovates in the concept of the game console, and it can control the game tasks by the actions of the body by using the balance instrument in the remote control handle. In Figure 4, player can use Wii to play baseball, and the handle of Wii equals to the baseball bat, and users' occasion and speed of flinging the handle will influence batting effects. Here, the handle of Wii becomes into the touching symbol, and it has been turned into certain special concept, i.e. the new life mode of body-building and amusement. The design concept of "the revolution of TV game" of Wii brand has wined many consumers' favors.

In conclusion, the interactive product symbol system is multi-dimensional, and when it is applied in the design practice, above sensibility interactive modes should be comprehensively used to form the integrated technology which can really express the sensible cognition and whole taste experience for certain product, and make finite symbol express infinite recurrence of language environment and product image and establish the special image of the product.

\subsection{Innovational semantic expression level analysis of the symbol system}

In the product design process, designers endow their thoughts and ideas to the product, which will form the interior meanings with the attributes of the product, and utilize many methods such as association, memory and metaphor in the semeiotics to endow the configuration, image symbol and language full of originalities to the product, which will produce a sort of communication of meaning between designers and consumers to make consumers fully understand the connotation of the product, and simultaneously, the communication of spirit culture will be implemented between designers and consumers through the symbol system, and the resonance between the product and the consumer will be produced to some extent through the emotion creation of the product and originality of the emotion value.

Taking the MrP little shamed reading lamp design of Propaganda brand in Figure 5 as the example, the switch is the forbidden zone, and if consumers don't touch it, the lamp will not be turned on. As consumers adapt this switch slowly, face it and abuse it, which is a sort of innovational semantics of the product. The facetious symbol of the product can express unique semantics, and added joking paronomasia and hinted ardor symbolic language can stimulate consumers' interior freedom sense and make them indulge themselves to the top of their bents.

\subsection{Symbolization expression level analysis of the symbol system}

The symbol of the product symbolizes certain culture, and its cultural value can be called as the subjective value. The symbolized semantics contains designer's thoughts and intentions, and designer could express the time culture, enterprise spirit and culture through the product. However, the symbol is not established at will, and it must possess common character with object, and make consumers could associate and deeply understand the cultural connotation according to their own knowledge background from the one to the other. Therefore, good innovational product should seek the optimal vision expression of the originality from the relations among signified, signifier and signifying, and exactly grasp the symbolized meaning of the symbol.

Taking the torch design of 2008 Beijing Olympics as the example, the torch adopted the symbolized technique of the symbol (seen in Figure 6). The "clouds lines" symbol of the torch is the recurrence of the traditional design element in the Chinese Huaxia Civilization, and it symbolizes the Huaxia Civilization of "born in same origin and being in harmony", and it can diffuse the harmonious culture and oriental civilization through the oriental spirit of "the world should be natural and the people should be full of interior and open-minded tolerance" and the image of the lucky clouds. Based on above meanings, the Legend Group which assumed the design of the Olympics torch created the brand product with national cultural characteristics.

\section{Some opinions about the product symbol system for enterprises to research and develop special brands}

To design the innovational product favored by consumers, designer should grasp following aspects of the product symbol system.

(1) The product symbol system should possess the character of difference. The existing symbol language should be abstracted, or distorted, or decomposed to form innovational symbol language. The innovational symbol system (including the trademark on the product, packaging symbol, the modeling and function indication of the whole produce, and the whole image concept) should make consumers can distinguish and remember the product, and be interesting to 
the brand, which will help the product to cultivate a passel of seed consumers and loyal consumers to extend the brand effect.

(2) The symbol semantics should possess the character of national culture. Designers should consider the national culture characters, and select the symbol language with the characters of national culture, and skillfully pick up and rebuild the symbol elements which could reflect the national characters to design the successful brand product which could map the connotation of the national culture. For example, the "lucky clouds torch" design in 2008 Beijing Olympics and the torch design in 2000 Sydney Olympics all possess bright national culture characteristics.

(3) The symbol should possess the affinity. The emotion communication is realized in the "friendly" communication process of the symbol, so the establishment of modern product brand should emphasize the "non-material design" and the emotional design.

(4) Designers should fully consider consumers' psychological acceptance. According to the "MAYA" threshold proposed by Raymond Loewy, i.e. the most advanced yet acceptable range, the design and utilization of symbol should consider the acceptable ability of the market and the public, and try to fulfill consumers' cultural background and psychological demands. In this way, consumers could distinguish and understand the symbolized meaning of the symbol, and the innovational brand could be favored and trusted by consumers, or else, the symbol language with more originality will run counter to consumers' psychologies.

(5) The thinking of symbol should possess the flexibility in the time space. The symbol should possess the character of variable thinking, and designer should flexibly utilize the symbol according with the characteristics of the times. As the symbol is continually developing with the variance of the time and space, designers should grasp the time and space applicability of the symbol, select or dig the symbol skillfully to exactly transmit the information and express the originality of the times.

\section{Conclusions}

Abundant product symbol system design with characteristics is the key for enterprises to strategically research and develop new product, and the key to start the innovational brand design. With the high-speed development of the information technology and the interactive integration of multiple subjects knowledge, and the design and development of the product symbol system is more and more emphasized by designers. How to enhance the symbol value of the product, and skillfully utilize the symbol system design to extend the brand effect has been the problem continually probed by designers. The research results are expected to be used for some references for the brand design.

\section{References}

Cao, Jujiang. (2007). Product Design Acceptance Based on Consumer. Packaging Engineering. No.2.

Cheng, Dinglv \& Jiang, Xiao. (2008). The Signified of Semiotics in Interaction Design. Art and Design. No.8.

Cupar (US). (2006). The Road of Interaction Design. Beijing: Publishing House of Electronics Industry. March, 2006.

Li, Ni. (2006). Research of Fun Product Design on the Basis of Semiotics. Packaging Engineering. No.5.

Mao, Jingjing \& Li, Shiguo. (2008). Interaction Design in the Sense Times. Consume Guide. No.1.

Wang, Hongjian \& Yuan, Baolin. (1997). The Concept of Art. Beijing: Higher Education Press. April, 1997.

$\mathrm{Xu}$, Shihu \& Liu, Lijuan. (2008). The Enlightenment of Traditional Graphic Symbol to the Furniture Design. Innovational Thinking and Practice. No.3.

Yang, Wenfa. (2008). Cultural Design of Product. Design and Culture. No.3.

Zhang, Linghao. (2006). The linguistic Meanings of Product. Beijing: China Architecture \& Building Press. 


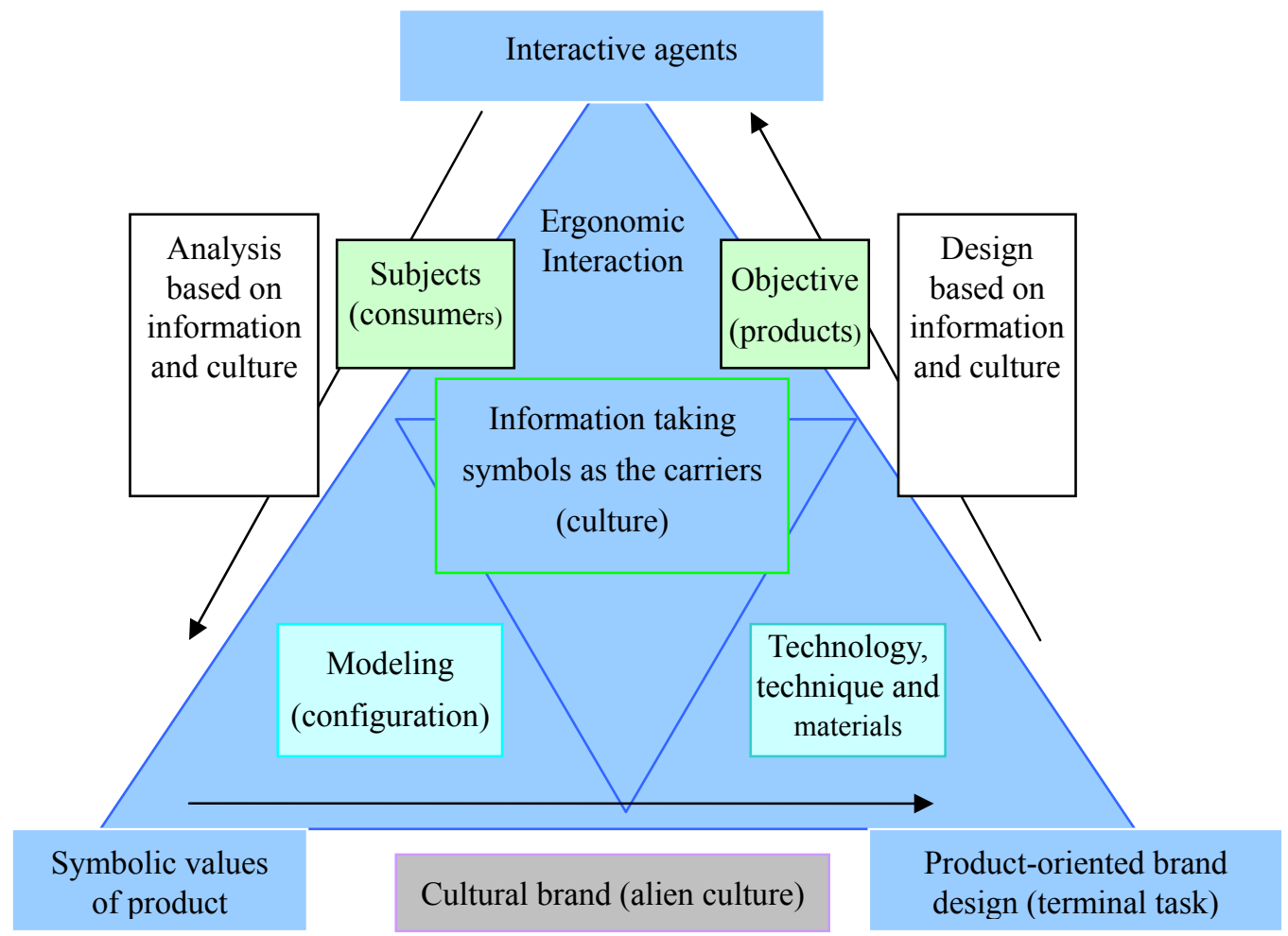

Figure 1. System Structure Relation of Interactive Product Symbols

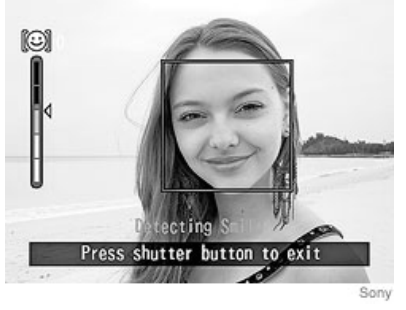

Figure 2. The "cyber-shot" Smile shutter Mode of Sony

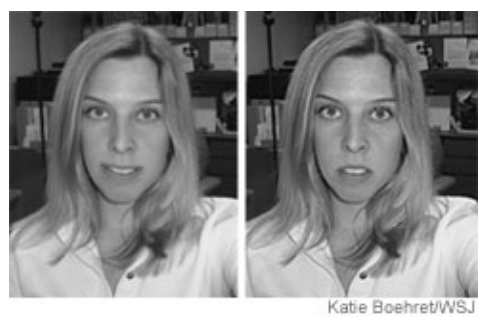

Figure 3. The Happen Face Retouching Function of Sony

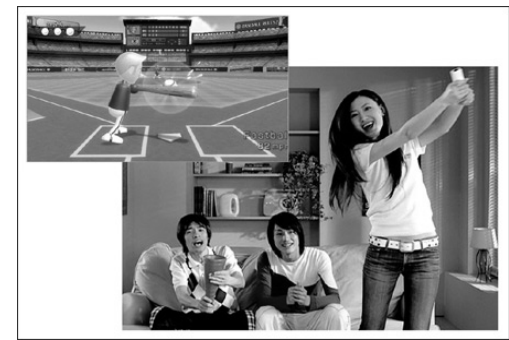

Figure 4. Touching Symbol Function of Wii Game Console Lever 

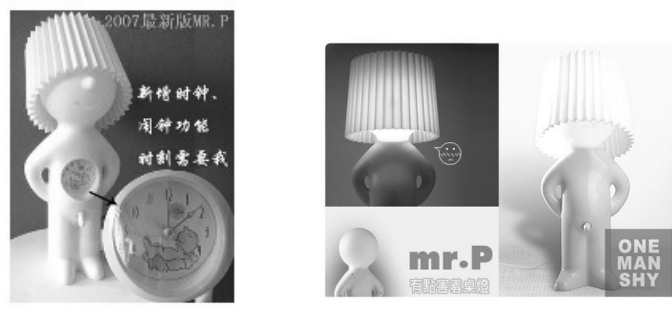

Figure 5. MrP Little Shamed Reading Lamp of Propaganda Brand

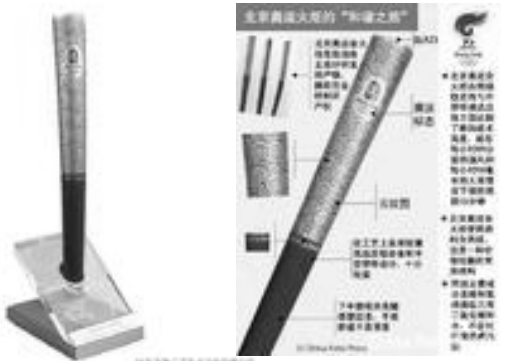

Figure 6. Design of Lucky Clouds Torch in 2008 Olympics 